\title{
Optimal control of a water distribution network in a supervisory control system
}

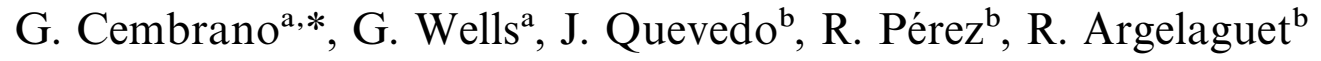 \\ anstituto de Robótica e Informática Industrial, Universidad Politécnica de Cataluña, Consejo Superior de Investigaciones Científicas, \\ Gran Capitán 2-4, 08034 Barcelona, Spain \\ ${ }^{\mathrm{b}}$ Departament Enginyeria de Sistemes, Automàtica i Informàtica Industrial, Edifici d'Automàtica - TR11, Universidad Politécnica de Cataluña, \\ Campus de Terrassa, Rambla Sant Nebridi, s/n, 08222 Terrassa, Spain
}

\begin{abstract}
This paper deals with the use of optimal control techniques in water distribution networks. An optimal control tool, developed in the context of a European research project is described and the application to the city of Sintra (Portugal) is presented.
\end{abstract}

Keywords: Optimal control; Water networks; Telecontrol; Nonlinear optimization; Modelling; Simulation

\section{Introduction}

Water networks are generally composed of a large number of interconnected pipes, reservoirs, pumps, valves and other hydraulic elements which carry water to demand nodes from the supply areas, with specific pressure levels to provide a good service to consumers. The hydraulic elements in a network may be classified into two categories: active and passive. The active elements are those which can be operated to control the flow and/or the pressure of water in specific parts of the network, such as pumps, valves and turbines. The pipes and reservoirs are passive elements, insofar as they receive the effects of the operation of the active elements, in terms of pressure and flow, but they cannot be directly acted upon.

A supervisory control system in a water network generally includes a telemetry system which periodically updates some information from a selected set of passive elements and from most of (ideally all) the active elements. This information is generally composed of pressure and flow readings, as well as status of the active elements, and it reflects the instantaneous operating con-

\footnotetext{
*Corresponding author. Tel.: + 34-3-401-5785; fax: + 34-3-4015750 .

E-mail address: cembrano@iri.upc.es (G. Cembrano).
}

dition of the water network. It also includes the mechanisms to actuate the active elements in the network and to control their performance. However, it is not always obvious how to derive appropriate control strategies for the active elements, in order to use the resources efficiently and to meet the specific pressure and flow needs in the whole network, at all times.

Optimal control in water networks deals with the problem of generating control strategies ahead of time, to guarantee a good service in the network, while achieving certain performance goals, which may include one or more of the following, according to the needs of a specific utility: minimization of supply and pumping costs, maximization of water quality, pressure regulation for leak prevention, etc.

The implementation of optimization procedures may prove an extremely efficient measure to contribute to the correct management of water resources and to reduce operational costs, as well as to improve the service to consumers, as has been shown in several water utilities around Europe. In the context of a European research project (WATERNET, ESPRIT IV, No. 22186), the authors have developed an optimal control tool integrated in a supervisory control system which can be used by a large class of water utilities. The whole system, including optimal control was demonstrated in a prototype of the water network of the Sintra (Portugal) area. In 
this paper, the concepts of optimal control in water networks are presented in Sections 1 and 2. Sections 3-5 deal with the mathematical statement, the complexity and the methods of solving the optimal control problem respectively and in Section 6, the application to the network of Sintra (Portugal) is presented. Section 7 summarizes the conclusions of this work and presents future research issues.

\section{On-line optimal control}

\subsection{Inputs from the network}

In a supervisory control system for a water network, the optimal control procedure interacts with the real network through the SCADA (Supervisory Control and Data Acquisition) system. It must receive information about the current state of the network, in terms of

- water volumes in reservoirs,

- status of pumps and valves,

- latest demand readings,

- pressure and/or flow readings at selected points.

In order to compute optimal control strategies ahead of time, the optimal control procedure must contain a prediction model to generate forecasts of the next future demands, based on a record of a number of past values thereof. A number of methods for demand prediction may be used as described elsewhere (Quevedo, Cembrano, Gutiérrez \& Valls, 1988; Griñó, 1991). In this work, the demand prediction model was obtained using the Fuzzy Inductive Reasoning Methodology, as described in López, Cembrano and Cellier (1996).

The demand information from the SCADA is used to update the past-demand record and a forecast of the demand in the next period is performed. The demand forecasts are usually obtained with a horizon of the order of one day. The hourly data is obtained by modulation with appropriate demand patterns for each day in the week.

\subsection{Optimization execution}

Additionally, the optimization module contains a hydraulic model (see Section 3.2) of the network which makes it possible to test the effect produced by a control action (flows through the active elements) on the network, in terms of

- water volumes in reservoirs,

- pressure and/or flow readings at selected points.

The optimal control procedure selects optimal strategies for the controllers of the active hydraulic elements, by searching in the space of possible controls and evaluating different alternatives, as described later in this

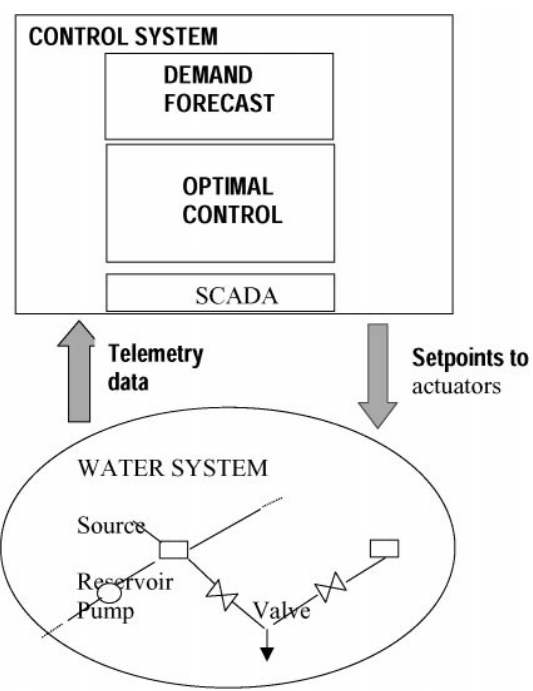

Fig. 1. Optimal control in water systems.

document. In water networks where storage in reservoirs must be planned ahead to meet the future demands with specific pressure levels, the optimization involves the generation of controller strategies over a time period, called the optimization horizon, which may be of the order of one day, at hourly intervals, for a common case of water distribution utility.

\subsection{Outputs to the remote units}

The optimal control values are sent through the SCADA as setpoints to the local controllers in the remote stations that regulate the active elements.

Optimization updates must be performed regularly during the day. The general scheme of interaction with the supervisory control system is shown in Fig. 1.

\section{The optimal control problem}

\subsection{Network description}

A convenient description of the dynamic model of a water network is obtained by considering the set of flows through the $m$ active elements as the vector of control variables

$u \in R^{m}$

and the set of $n_{r}$ reservoir volumes monitored through the SCADA as a vector of observable state variables

$r \in R^{n_{r}}$

Additionally, in order to complete the state description of the network, it may be necessary to define a vector

$q \in R^{n_{q}}$ 
containing the values of flow at $n_{q}$ specific passive elements, which cannot be directly measured through the SCADA and a vector

$p \in R^{n_{p}}$

containing the values of pressures at $n_{p}$ specific nodes, not monitored through the SCADA.

Vectors $p$ and $q$ may be obtained from $r$ and $u$ through explicit or implicit, generally nonlinear, equations expressing the hydraulic relationships which couple head and flow. Well-known approximations of these hydraulic relationships exist for different types of hydraulic elements. For pipes, for example, the Colebrook-White, the Darcy-Weisbach or the Hazen-Williams equations (see e.g. Isaacs \& Mills, 1981; Jeppson, 1983; Quevedo, Cembrano, Casanova \& Ros, 1987) have been widely used in network flow and pressure calculations. Similarly, a good approximation of the head-flow relationship for pumps may be found in Anderson and Powell (1999) as well as in appropriate manufacturer's specifications. For valves, head-flow equations, according to their characteristics and their functionality are usually supplied by the manufacturers. Some of the most relevant examples of these equations may be found in Greco (1997). Hence,

$p=h(r, u, d)$,

$q=g(r, u, d)$,

where $d \in R^{n_{d}}$ is a vector of stochastic disturbance containing the values of the demands at the $n_{d}$ consumer nodes in the network.

Since the model is used for predictive (optimal) control, $d$ will generally be a vector of demand forecasts, obtained through appropriate prediction models (see Section 2.1).

An augmented state vector is defined as

$x \in R^{n}$

$n=n_{r}+n_{q}+n_{p}$

where $x$ contains the $n_{r}$ values of reservoir volume vector $r$, the $n_{q}$ values of the flow vector $q$ and the $n_{p}$ values of the pressure vector $p$.

\subsection{Dynamic model}

The dynamic model of the network may then be written, in discrete time, as

$x^{k+1}=f\left(x^{k}, u^{k}, d^{k}\right)$,

where $f$ represents the mass and energy balance in the water network and $k$ denotes the instantaneous values at sampling time $k$.

\subsection{Performance index}

The control actions are generated by optimizing a performance index, i.e. a mathematical expression of the desired exploitation goals.

In general, a performance index may contain one or more of the following terms:

- The cost of water supply, treatment and elevation $C_{u}$, which is a function of the flows in the $m$ active elements contained in vector $u$.

- Artificial costs related to pressure regulation, expressed in functions $C_{p}$ of the pressures in $n_{p}$ specific key nodes of the network, contained in vector $p$.

- Artificial costs related to flow regulation and/or water quality, $C_{q}$, expressed as function of the flows in $n_{q}$ specific pipes of the network, contained in vector $q$.

Then, a general performance index may be expressed as follows: For an instantaneous evaluation,

$P^{k}=\sum_{i=1}^{m} c_{u}^{k}\left(u_{i}\right)+\sum_{j=1}^{n p} c_{p}^{k}\left(p_{j}\right)+\sum_{l=1}^{n q} c_{q}^{k}\left(q_{l}\right)$,

where $P$ is the instantaneous value of the performance index and $k$ refers to the values of the instantaneous values at the $k$ th time period.

For an evaluation over the entire optimization horizon, the performance index is

$J=\sum_{k=0}^{N-1} P^{k}$,

where $N$ is the optimization horizon (in a number of sampling periods).

\subsection{Constraints}

The performance optimization must take into account that a number of important constraints exists:

- On the control variables, such as the flows across the active hydraulic elements. Pumps and valves have specific ranges of flow for correct operation.

$u \in U, \quad U \subset R^{m}$

- On other variables, such as the levels in the reservoirs. These include capacity and operational limits on water minimum and maximum allowed levels. Similar constraints may be in order in the selected $n q$ pipes and the $n p$ pressure measurement nodes. Note that the optimization can only be performed in the space of controls while these constraints are expressed in terms of other variables, which cannot be actuated directly.

$r \in S, \quad S \subset R^{n_{r}}$,

$q \in Q, \quad Q \subset R^{n_{q}}$,

$p \in H, \quad H \subset R^{n_{p}}$. 
The optimization process consists of searching the space of admissible control actions to obtain the minimum (or maximum) value of the performance index, while producing only admissible states of the network.

It is important to remark that the performance index can only be minimized with respect to the control variables (flows across active hydraulic elements), since these are the only variables which may be acted upon. However, the value of the index also depends on variables that are not directly controlled, but must be evaluated for each control action. Similarly, the contraints apply both on directly controlled variables and on other variables related to passive elements, which cannot be operated directly.

In many practical cases a distinction can be made between "hard" and "soft" constraints, the former being those which must be fulfilled strictly and the latter, those which may be violated to some moderate extent. In particular, the operational levels of reservoirs, the minimum-pressure and the flow-range constraints may usually be defined so that only soft bounds are imposed. In this case, the optimization may proceed by augmenting the performance index with penalty terms which impose the approximate fulfilment of the soft constraints, using Lagrange multipliers (see e.g. Minoux, 1986).

\section{Complexity of the optimal control problem in water networks}

The computational complexity of the optimal control problem in water networks is determined by a number of important factors in the real network and in the modelling assumptions. The main factors and their influence on the optimization complexity are outlined below.

\subsection{Size}

The size of the optimization problem depends on the number of active hydraulic elements to be optimized, on the number of passive elements which reflect the state of the network, such as reservoirs and pressure- or flowevaluation nodes, but also, most importantly, on the optimization horizon. Instantaneous optimization problems pose few computational difficulties, but dynamic optimization problems over 24 or more hourly intervals, which are common in many water utilities in Europe, impose a very high computational burden in non-trivial networks. This is so because of the combinatorial nature of the problem of searching the space of controls for a number of interacting active elements in a 24-stepahead horizon.

\subsection{Type of hydraulic elements involved}

The type of hydraulic elements affects the design of strategy optimization programs insofar as it determines the type of variables to be used to express flows and pressures. This is important since very efficient methods exist for optimizing vast classes of problems involving continuous variables and continuously differentiable functions, especially because it is possible to use gradient-based searchs. Conversely, the methods of integer programming required to optimize problems with integer or binary variables are mainly of combinatorial nature and they cannot make use of the gradient concepts. They are, therefore, usually slow and not necessarily guaranteed to converge. For this reason, it is often preferred to work with continuous approximations of the values of the discrete variables involved in the optimization, as follows:

- Valves: They allow any value of the flow in its operational range. Therefore, continuous variables can be used to represent the value of the flow through a valve.

- Fixed-speed pumps: For a specified value of the downstream pressure or the head increase, only one value of the flow is possible according to the characteristic equation of the pump. Therefore, the instantaneous value of the flow through a pump cannot be realistically modelled using continuous variables. However, the volume of water through a pump in a specified period (e.g. hourly periods, as required for the dynamic optimization) may attain any desired (feasible) value through pulse-width modulation, i.e., by operating the pump only a portion of the period.

- Batteries of fixed-speed pumps: Utilizing a number of fixed-speed pumps usually allows for a modulation in the flows, which makes it easier to model the flow as a continuous variable. Additionally, continuousvariable optimization methods may be modified to produce sub-optimal solutions which are close to integer numbers. An example of one such approach is given in Section 6.2.4.

\subsection{Type of network description}

The effect on the network of each candidate control strategy tested by the optimization must be evaluated using the network description and hydraulic couplings stated in Eqs. (1)-(3). The complexity of these equations will obviously influence the time required by the optimizer to evaluate each candidate control action.

- Flow-only models and, in particular, those models where only the control variables are required to compute the change in the state of the network produced by a control action allow a very fast evaluation of control actions.

- Flow and pressure models, where a number of pressures and/or flows in passive elements are required to compute the change in the state of the network produced by a control action, impose longer execution times for each test. The problem is easier when explicit 
equations exist to compute the pressures and/or flows in passive elements, in terms of the control variables. If this is not the case, an iterative simulation procedure must be run for each evaluation of a control action. This condition imposes considerably longer execution times.

\subsection{Type of optimization equations}

The availability and computational complexity of optimization methods varies according to the form of the equations involved. In particular, for continuous-variable problems:

- Problems with linear performance indexes and linear constraints have fast and efficient solution methods based on the SIMPLEX method.

- Problems with quadratic performance indexes and linear constraints have fast and efficient methods based on gradient techniques, which are guaranteed to converge in a number of iterations equal to the dimension of the optimization space.

- Problems with other nonlinear convex performance indexes and linear constraints may be solved using the same methods as above, without the guarantee of the number of iterations for convergence.

- More general problems with convex performance indexes and nonlinear constraints defining a convex space may be solved with gradient-based techniques efficiently.

- Nonconvex problems pose serious difficulties, as there is no guarantee that only one extremum (minimum or maximum) exists. In these cases, random- and heuristic-search methods may be used.

- For integer or binary-variable systems, the optimization problems are of a combinatorial nature. Search and pruning methods are available, such as the branch-and-bound algorithms, which are efficient in some specific cases with a moderate dimensionality.

\subsection{Topology}

The topology determines how an action in a certain element of a water network affects the rest of it. For example, in some simple tree-like networks an action at the end of a final branch may not affect the rest of the network at all, while in a mesh-like network, a more global influence of the actuation of most of the hydraulic control elements is to be expected. This situation is usually an important factor to be taken into account for the selection of more or less de-centralized schemes for the supervisory control system in general and for the control strategy optimization in particular. Star-structure water networks generally contain one large source (usually with an associated reservoir) from which water is supplied to a number of local small distribution areas, each of which may contain at most one reservoir, as shown in Fig. 2.

The special characteristics of this network allow the use of spatial decomposition techniques for optimization, which make it possible to compute a solution to a large complex problem through the iterative solution of a number of simple problems, see e.g. Coulbeck (1977). Note, in particular, that the sense of the flows is fixed, from the central source to the demands.

Tree-structure water networks generally have one large source (and usually, an associated reservoir) from which water is supplied to a small number of intermediate locations, where it is stored and/or pumped to the demand nodes, as shown in Fig. 3.

Note that here too, the sense of the flows is defined, from the source to the demands.

A cascade water network is a special case of a treestructure network where there is only one branch, as shown in Fig. 4.

A mesh-structure network usually contains several sources (e.g. with associated reservoirs) and is highly interconnected so that the demands can be supplied from more than one source and, in general, with different operation patterns, so that the sense of the flows is not fixed in some of the valves and pipes (see Fig. 5).

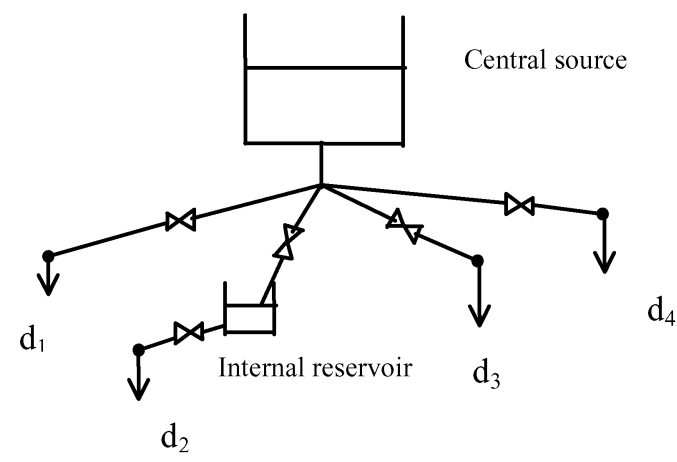

Fig. 2. Star-structure water network.

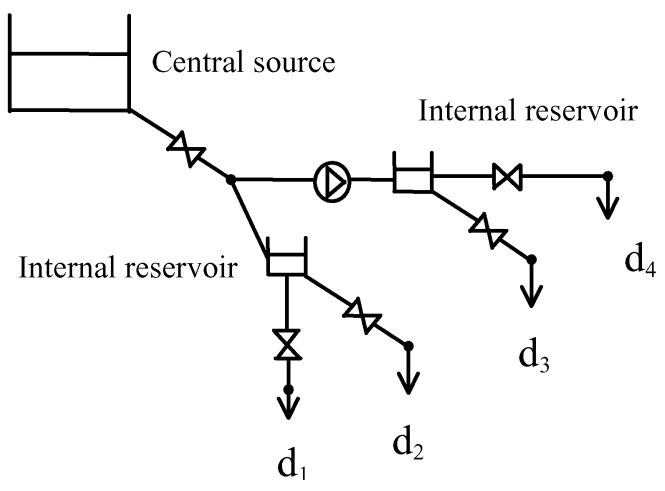

Fig. 3. Tree-structure water network. 


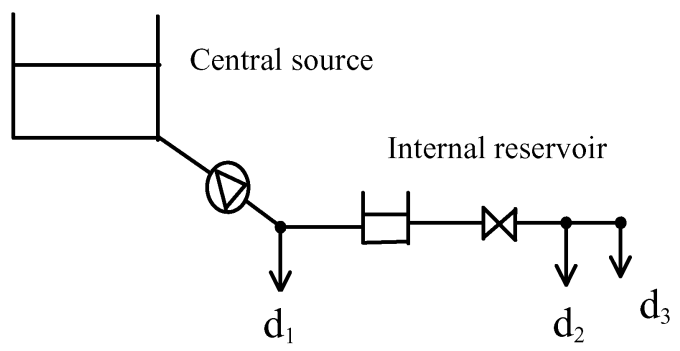

Fig. 4. Cascade structure water network.

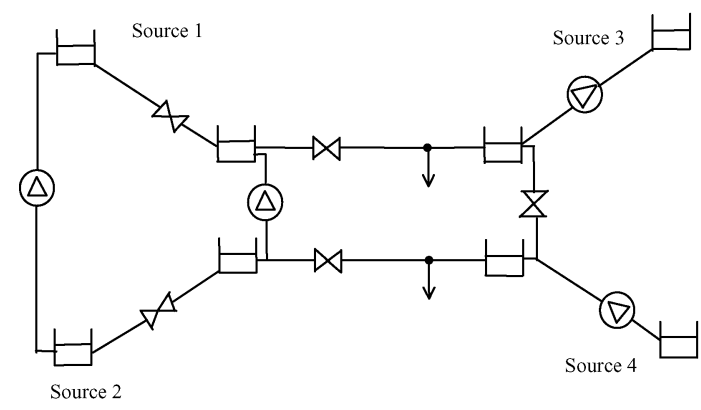

Fig. 5. Mesh-structure water network.

\section{Solving the optimal control problem}

\subsection{State of the art}

A number of large European utilities already utilize optimization programs to generate $24 \mathrm{~h}$ strategies ahead in time to minimize costs while maintaining a good service to consumers. Several types of optimization procedures have been used, but most of them employ either dynamic programming or convex nonlinear programming techniques. In most cases, the size of the optimization problems involved makes it necessary to derive special methods, since the direct application of the techniques would not have produced acceptable computation times.

In the 1970s, Fallside and Perry (1975) derived a hierarchical price-decomposition algorithm for the optimization of the Severn-Trent Water Authority network. The solution of the subproblems used a linearization around an operating point to reduce the mathematical complexity. The method was implemented in the Cambridge area, but it did not achieve completely satisfactory results in the long run.

A more general scheme of hierarchical-decomposition optimization in water networks was proposed by Coulbeck (1977) and tested in simulation for other UK networks.

In Barcelona, Solanas (1974) derived a successive approximation dynamic programming algorithm for the Barcelona network, which was later improved and tested in simulation (Solanas \& Montoliu, 1988).
In the US, Murray and Yakowitz (1979) developed the differential dynamic programming techniques which allowed the use of the dynamic programming approach to the optimization of a relatively small simulated water network.

In the 1980s, one of the most relevant applications was the real implementation of the interaction prediction method developed by Carpentier (1983), in the network of Paris Sud of the French Company Lyonnaise des Eaux. This method was also based on the use of dynamic programming techniques and it made specific use of the star structure of the distribution network.

Later, a nonlinear programming technique was developed for the Barcelona network, which made use of optimal control concepts (Cembrano, Brdys, Quevedo, Coulbeck \& Orr, 1988), and an improvement thereof was implemented in the real network in 1990 (Griñó \& Cembrano, 1991). The Barcelona network is highly interconnected and has a mesh structure, which makes it inefficient to use decomposition techniques.

In the 1990s, Brdys (1992) considered the problem of optimizing the configuration of fixed-speed pumps in the water networks of Leicestershire and Yorkshire. He studied a class of cascade hydraulic systems in two cases: with weak and with strong hydraulic interactions. Spatial and time decomposition are used in combination with branch-and-bound mixed-integer programming techniques to solve the optimization problems of these two areas. The method was implemented successfully by the Yorkshire Water Authority (Brdys \& Ulanicki, 1994).

Most recently, a number of European joint research and development projects have attempted to solve more general classes of optimization problems and to produce tools applicable to a variety of water utilities throughout Europe. For instance, the CLOCWiSe (INNOVATION Programme) project dealt with the general problem of optimized scheduling of water management and network maintenance, through the use of Constraint Logic Programming, producing a specific tool applicable to many water control systems.

The WATERCIM project (ESPRIT Programme) paid special attention to the concept of integrating different water management tools, which had so far been used independently, with no information sharing.

The WATERNET project, which has produced this work, involved the development and dissemination of advanced techniques and tools for water management and planning, including modelling, learning and knowledge acquisition, simulation and state-estimation, optimal control, quality analysis, and distributed-informationsystem design.

In the area of optimization, the project goal was to demonstrate the benefits of introducing optimization in a variety of water utilities. Additionally, the WATERNET optimal control procedure incorporates aspects that had so far been avoided because of their 
computational complexity. This is the case of networks with hydraulic models involving flows and pressures for the optimization and the interaction with the state estimation and alarm management procedures to validate and correct optimization results.

\subsection{The WATERNET optimal control module}

\subsubsection{Structure}

The WATERNET optimization solver relies on a powerful commercial optimization tool (CONOPT), which can efficiently deal with linear or nonlinear cost functions and constraints. It incorporates several optimization procedures and it can provide information on the optimization process, as required by the user, with different degrees of detail. The WATERNET optimization module also incorporates a commercial interface to the CONOPT program (GAMS, 1997) which makes it simpler for a user who is knowledgeable in optimization to input the definition of the problem to the CONOPT program.

In addition, a higher-level user interface has been developed and added, so that it is simple for a water facility user to introduce a water optimization problem, with no previous knowledge in optimization and then to run the optimization and analyze the results very easily. This combination constitutes a powerful tool for the optimization of a very broad class of water systems.

\subsubsection{The problem statement interface}

The problem statement interface allows the user to input the optimal control problem. In particular, it is possible to create and visualize the network, through the network window (Fig. 6), and to define a performance index, as well as to impose constraints on the maximum and minimum values of the reservoir volumes, pump flows, valve openings, pipe flows and node pressures, through the Edit constraints window (Fig. 7).

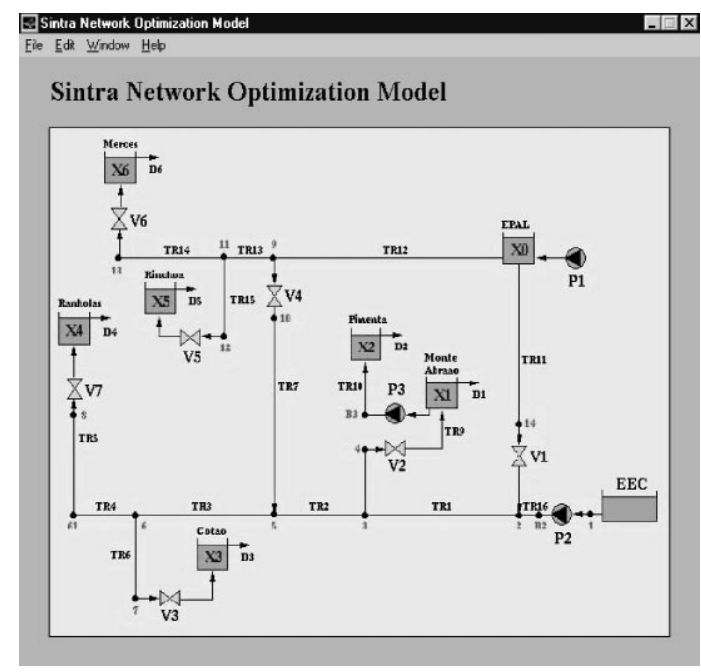

Fig. 6. The network window.

\subsubsection{The result presentation and analysis interface}

This interface shows the results of the optimization process (Figs. 8 and 9), in terms of strategies for the control elements and evolution of the reservoir volumes. Additionally, it is possible to visualize details of the optimization execution log.

\subsubsection{Integration with other water management tools}

The WATERNET optimal control module is executed in cooperation with other water management tools, corresponding to other modules in the WATERNET project. In particular, it interacts with the Supervision system, and with the Simulation, Learning and Quality Control subsystems.

At a fixed time everyday the optimization is started according to the scheme shown in Fig. 10. First of all, it retrieves the current status of the network - reservoir volumes, pump status, valve ratios - from the Supervision Subsystem. From the Learning Subsystem, it gets the demand-forecast results. Once the optimization procedure is run, it also interacts with the simulation module for the validation of a provisional set of control

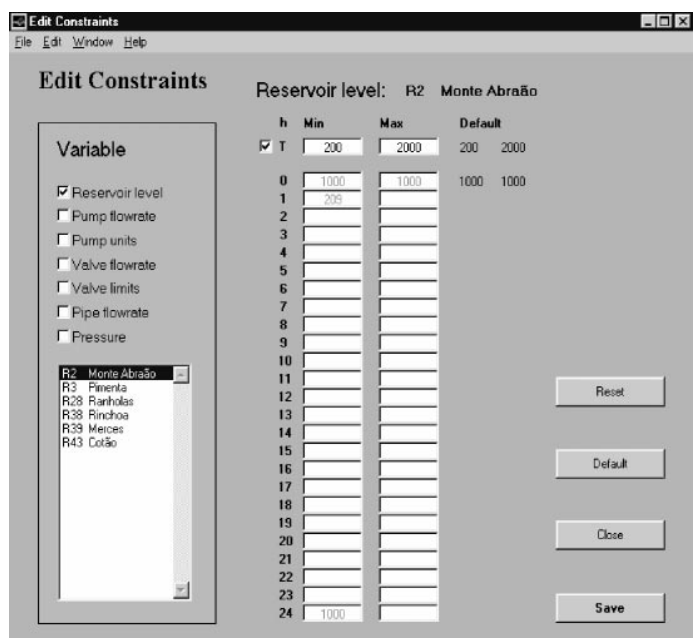

Fig. 7. Edit constraints window.

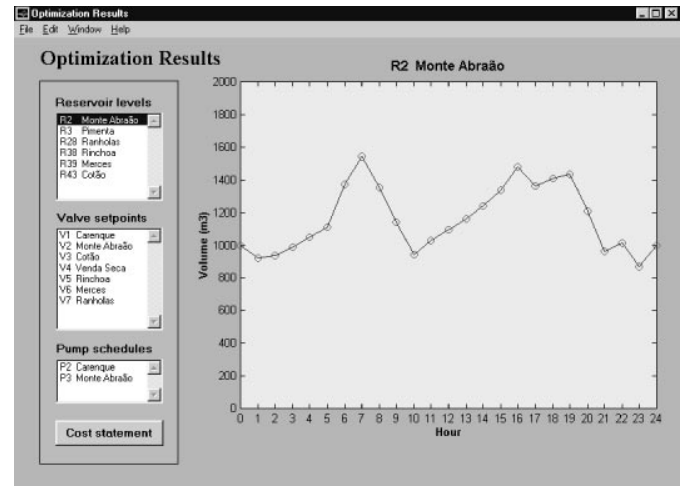

Fig. 8. The optimization results window showing a reservoir evolution. 


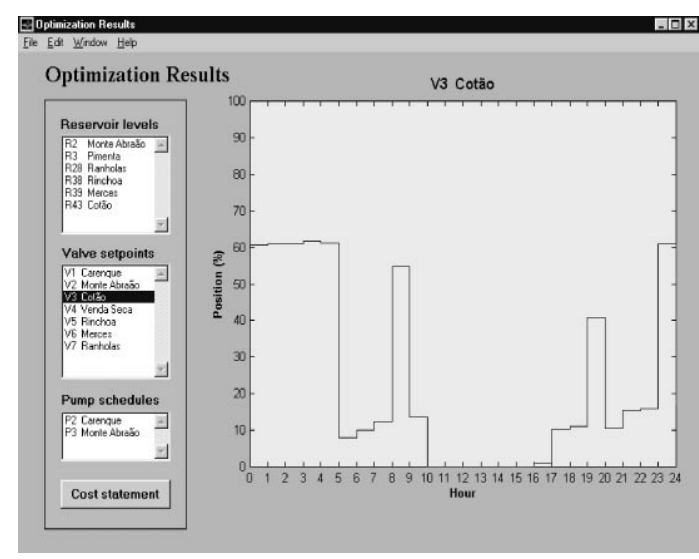

Fig. 9. The optimization results window, showing a valve strategy.

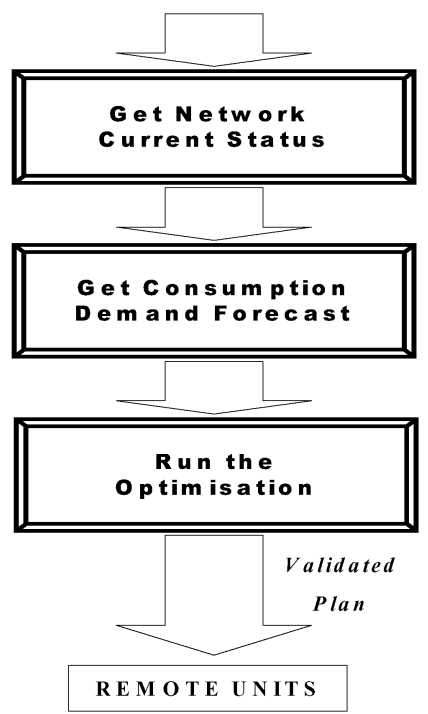

Fig. 10. Optimization strategy.

strategies in a more detailed network. It is not until the results of the simulation in a detailed network appear reasonable to a human operator that the strategies are considered correct. Similarly, the provisional control strategies are analyzed by the Quality Control module for consistency with the quality requirements and reoptimizations must be run with new conditions, expressed in constraints on the maximum or minimum flows to be taken from the sources, until the quality standards are met. Only then are the optimization results considered final.

\section{A case study: The Sintra network}

\subsection{Description of the network}

SMAS-SINTRA is a public company responsible for the drinking water network of the district of Sintra in Portugal as well as the wastewater service. The water distribution network spreads in a wide area, forming a total of $900 \mathrm{~km}$ of main pipes covering almost all the western area of the Lisbon region. They serve a satellite zone of Lisbon comprising residential users who commute to Lisbon to work (approx. 100000 inhabitants).

The network model includes 167 pipes, 204 nodes, 14 pumps and $11 \mathrm{ON}-\mathrm{OFF}$ valves. A large number of small-capacity reservoirs exists and the elevation varies greatly within the network. The difference between the extreme reservoirs is $277 \mathrm{~m}$. There are two main water supplies, related to pumping stations UB1 and UB2 in Fig. 6.

Because of its size, its mesh topology and the need for a flow-and-pressure hydraulic calculation, this model is unmanageable for optimization purposes. In order to produce a simplified version of the original model, the following criteria were used:

- Eliminating small-capacity reservoirs.

- Eliminating small-diameter pipes, while preserving the ontiguity of the network.

- Keeping all the active elements and any other elements supervised by the SCADA.

From this reduction, the operative model shown in the display of Fig. 6 is generated. It contains: 7 reservoirs, 2 pumping stations, 7 valves, 11 pipes and 6 consumption nodes and has the agreement of SMAS experts. The validity of the simplified model was verified by simulation. The pressures and flows in all the elements of the simplified model were computed by simulation on both (original and simplified models). It was confirmed that the flows and pressures computed with both models were not significantly different. Details of these experiments are reported in Pérez, Cembrano and Quevedo (1998).

\subsection{Statement of the optimal control problem}

\subsubsection{Optimization goals and performance index}

The optimization goal in the Sintra network is the minimization of cost in acquisition and pumping operations. For each pump $i$ and for each time period $k$, this is expressed as

$J^{k}(i)=J_{N L}^{k}(i)+J_{L}^{k}(i)$

where $J_{N L}$ is the hourly pumping cost, expressed as a nonlinear function of the flow through the pump and $J_{L}$ is the acquisition cost, expressed as a linear function of the flow:

$J_{N L}^{k}(i)=\alpha^{k} \Delta t \frac{\gamma \Delta h_{i}^{k} u_{i}^{k}}{\eta_{i}^{k}}$,

$J_{L}^{k}(i)=\lambda^{k} u_{i}^{k} \Delta t$,

where $i$ refers to the $i$ th pump station, $k$ refers to the $k$ th time period, $\alpha$ is the electricity tariff per energy unit, $\Delta \mathrm{t}$ is the sampling period, $\gamma$ is the specific weight of water, $\Delta h$ 
is the pump head increase, $u$ is the pump station flow, $\eta$ is the efficiency of the pump and $\lambda$ is a constant which expresses the cost of acquisition per unit volume. Then, the total cost is computed as a summation over the optimization horizon and over all the pumps in the network

$J=\sum_{k=0}^{N-1} \sum_{i} J_{N L}^{k}(i)+J_{L}^{k}(i)$

where $N$ is the optimization horizon expressed in number of sampling periods. In this case, the horizon is equal to 24 hourly intervals.

\subsubsection{Constraints}

The constraints are related to the mass balance in the whole network, the head-flow relationship in pipes and valves, the operational limits on the reservoir volumes, and a pressure limitation at one junction node.

\subsubsection{Initial and final conditions}

The initial condition of the network is read from the SCADA system and is expressed in terms of the reservoir levels at time 0 .

In this case study, a final condition is imposed as well, stating that the final volumes in the reservoirs should be the same as the initial ones. This condition makes sense when a $24 \mathrm{~h}$ periodic operation of the reservoirs is sought.

\subsubsection{Obtaining discrete values for pump setpoints}

At each pumping station $i$ of the network, there are a number of identical pumps in parallel, with equal nominal flow, $q_{i}$. Let $s$ be an m-dimensional vector that contains, in each component $i$, the number of pumps switched on at pumping station $i$. Then, at each sampling interval $k$, the value of the total flow through this station (corresponding to the $i$ th component of vector $u$ ), may be expressed as

$u(i)^{k}=s(i)^{k} q_{i}$,

so that optimizing $s$ is equivalent to optimizing $u$. Integer values of $s$ correspond to setpoints indicating a certain number of pumps switched on during the corresponding time interval. Non-integer values may be translated into setpoints corresponding to switching on a number of pumps for a fraction of the sampling interval.

Since it is important to avoid too frequent switching operations for the pumps, it is preferable to obtain values which are close to integer numbers for the components of vector $s$. In order to achieve this, a penalty function $J_{\text {art }}$ is added to the performance index. It penalises the flow values that do not correspond to an integer number of pumps on:

$J_{\text {art }}^{k}=\sum_{i=1}^{m} w\left(1-\cos 2 \pi s(i)^{k}\right)$,
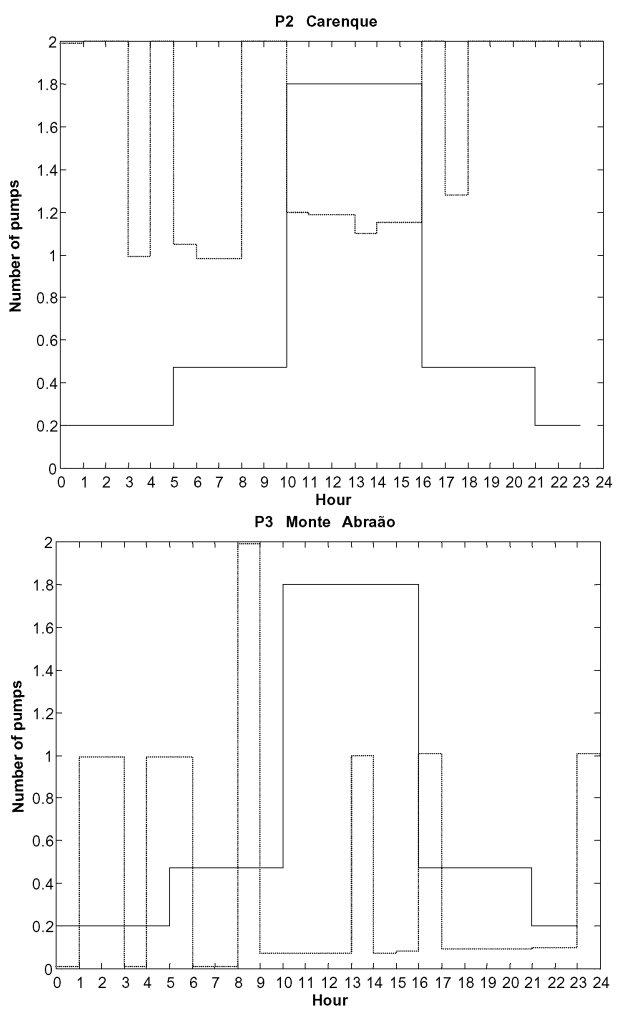

Fig. 11. Setpoints for the pumping stations.

Table 1

\begin{tabular}{lrc}
\hline Strategy & Manual & Optimal \\
\hline Water source 1 & 1425 & 1573 \\
Water source 2 & 2068 & 1188 \\
Pumping Carenque & 618 & 608 \\
Pumping Monte Abraao & 39 & 19 \\
Total & 4150 & 3388 \\
\hline
\end{tabular}

where $w$ is a penalty weight. This function takes minimum (zero) value when the values of $s$ are close to integers, so that the set-points (expressed in number of pumps on) can be approximated to the nearest integer for the simulation and real implementation.

\subsubsection{Optimization method}

The optimization method is a generalized reduced gradient method, first suggested by Abadie and Carpentier (1969), implemented as a part of the CONOPT library, which can cater for the nonlinear performance index and constraints. It starts by finding a feasible solution; then, an iterative procedure follows, which consists of:

- finding a search direction, through the use of the Jacobian of the constraints, the selection of a set of basic variables and the computation of the reduced gradient, 
R2 Monte Abraão

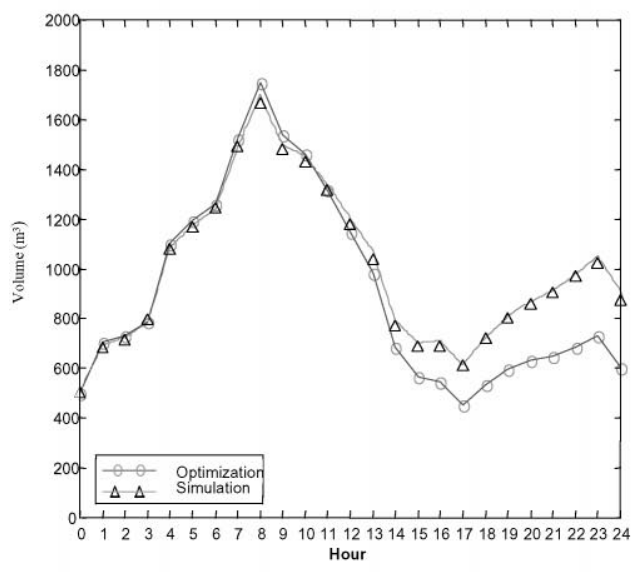

R3 Pimenta

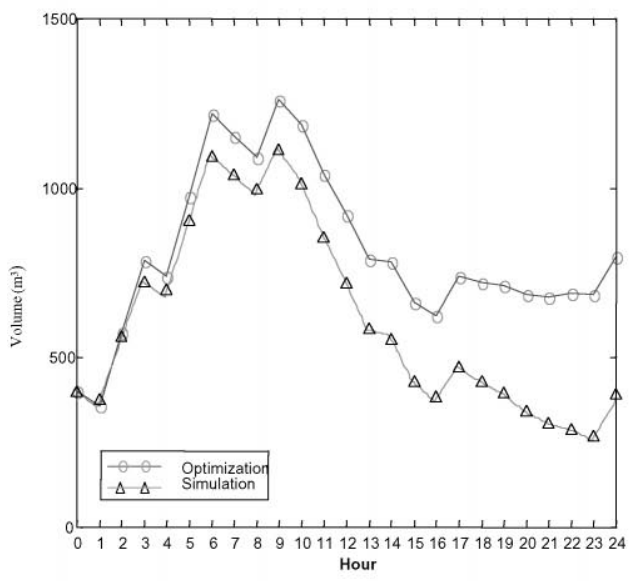

R43 Cotão

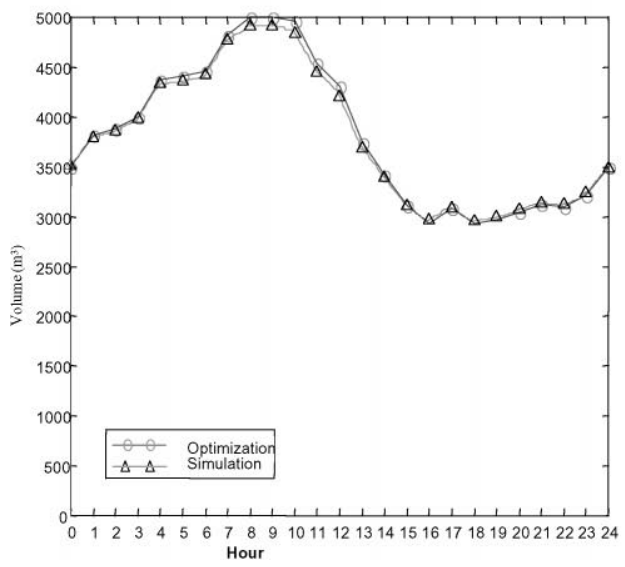

R28 Ranholas

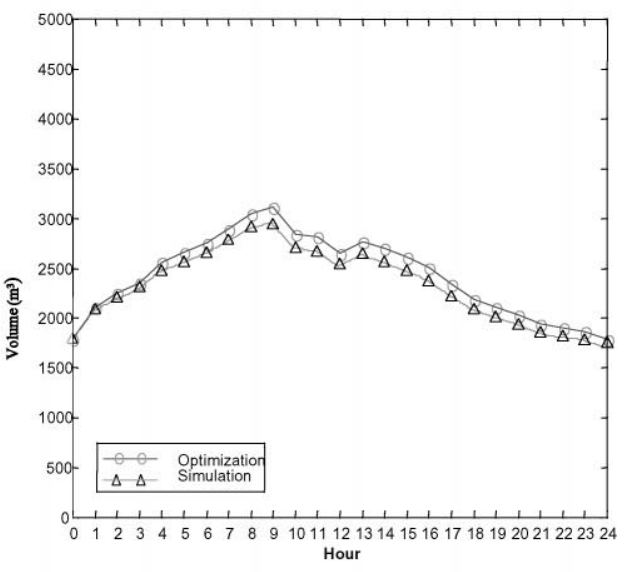

R39 Merces

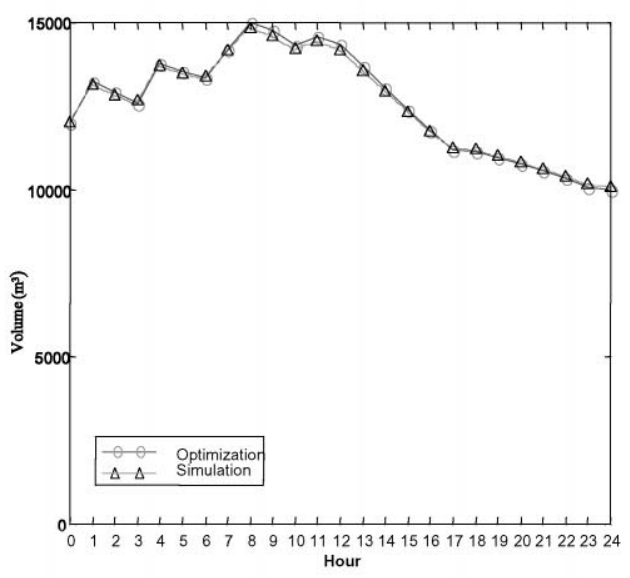

R38 Rinchoa

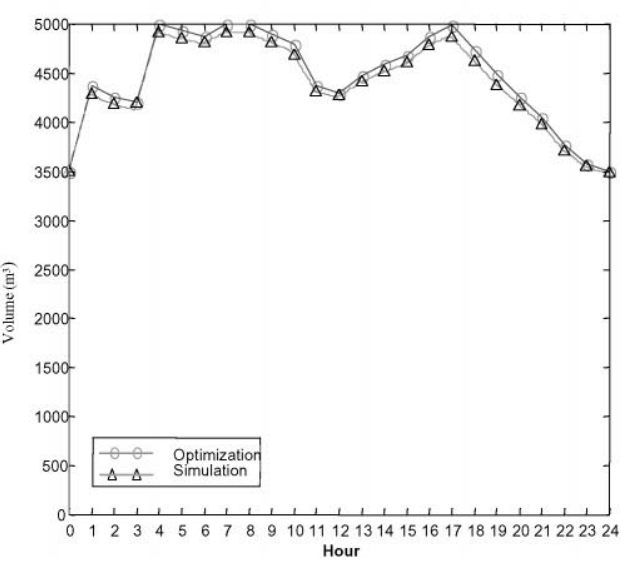

Fig. 12. Reservoir levels, optimization vs. simulation.

- performing a search in this direction, through a pseudo-Newton process

until a convergence criterion is met. A description of the algorithm and its implementation may be found in GAMS (1997). A more detailed version thereof is reported in Drud $(1985,1992)$.

\subsection{Results}

In this section, an example of the optimal control results is presented for one day of operation. Firstly, an optimization is run; then, a simulation is carried out in order to validate the strategy on a more detailed network model and, finally, the cost of the manual strategy is 
compared with the optimal one, computed through the optimization procedure.

Fig. 11 shows the optimal control strategy for two pumping stations. As can be seen, the pumps are ON mostly (dotted line) when the electrical tariff is low (solid line). However, since some of the demands are very hard to meet, the pumps must occasionally work with an expensive tariff.

Fig. 12 shows the evolution of the reservoirs, according to the simplified and the detailed models. In both cases, it may be observed that the evolution is satisfactory, since no extreme (overflowing or empty) conditions are reached while satisfying all the demands.

For illustration, the results of the optimal control procedure are compared to the actual manual strategy which was used on one day (specifically December 15, 1997).

In Table 1 the costs in ECU of the manual, and optimized strategy are compared.

It is important to remark that the numeric values of this comparison must only be considered orientative, because the optimal control results are based on models that are definitely a representative of the actual hydraulic system, but their parameters have not yet been thoroughly calibrated, whereas the results of the manual strategy are retrieved from the historical data of the real network. In any event, it may be appreciated that, by implementing the optimal strategy, a considerable saving might have been achieved. This includes, most importantly, a water cost reduction and an additional energetic cost minimization, due to a good pump scheduling.

\section{Conclusions}

Optimal control in water networks is an efficient means of scheduling water transfer operations to achieve management goals, such as cost minimization, quality improvement, pressure regulation, etc. So far, only very few water utilities have incorporated optimal control procedures in their supervision systems.

The optimal control module described in this paper is applicable to a broad class of water networks and incorporates a number of improvements over previous optimization applications. In particular, it is possible to solve optimal control problems in networks where the hydraulic model includes the nonlinear coupling between flows and pressures. Additionally, although the flows are treated as continuous variables, a solution that is close to the discrete possible setpoints of the pumps is obtained. Furthermore, the interaction with other water management tools, such as the simulation and the quality control programs, guarantees that feasible and reliable solutions are produced.

The case study presented in the paper shows that significant savings may be achieved by using the optimal control procedures to compute the strategies for pumping and water transfer operation, as compared to the actual strategies computed intuitively by an operator. This is the case in a variety of water distribution systems, where the structure, the size or the complexity of the hydraulic interactions make it hard for a person to produce optimal control strategies.

Future research issues on this subject include the calibration of water network models, the improvement of the methods to cater for other water management needs, such as the minimization of switching operations in pumps and valves and the improvement of the techniques for obtaining integer strategies for the pumping operations.

\section{Acknowledgements}

This work has been partly funded by the Commission of the European Communities (ESPRIT-IV No. 22186) and by the Research Committee of the Generalitat de Catalunya (group SAC, ref. 1997AGR00098). The authors are members of CERCA (Association for the Study and Research in Automatic control) and of LEASICA (Associated European Laboratory on Intelligent Systems and Advanced Control). The contribution of Messrs. Carlos Martins Nunes and Jorge Vilala of SMAS (Servicios Municipalisados de Agua de Sintra, Portugal) in supplying data and validating the experiments is gratefully acknowledged.

\section{References}

Abadie, J., \& Carpentier, J. (1969). Generalization of the Wolfe reduced gradient method to the case of nonlinear constraints. In R. Fletcher, Optimization (pp. 37-47). New York: Academic Press.

Andersen, J. H., \& Powell, R. S. (1999). The use of continuous decision variables in an optimising fixed speed pump scheduling algorithm. In R. Powell, \& K. S. Hindi, Computing and control for the water industry. UK: Research Studies Press.

Brdys, M. A. (1992). An algorithm for optimal scheduling of a class of cascade water supply systems. Optimal Control Applications and Methods, 13, 265-287.

Brdys, M. A., \& Ulanicki, B. (1994). Operational control of water systems: Structures, algorithms and applications. UK: Prentice-Hall International.

Carpentier, P. (1983). Decomposition et aggregation dans la conduite optimale d'un grand reseau de distribution d'eau, Ph.D. thesis, Ecole Nat. de Mines de Paris.

Cembrano, G., Brdys, M., Quevedo, J., Coulbeck, B., \& Orr, C. H. (1988). Optimization of a multireservoir water network using a conjugate-gradient technique. In A. Bensousan, \& J. L. Lions, Lecture Notes in Control and Information Science, Vol. 111. Berlin: Springer (pp. 987-989).

Coulbeck, B. (1977). Optimization and modelling techniques in dynamic control of water distribution systems. Ph.D. thesis, University of Sheffield, Sheffield, UK.

Drud, A. (1985). A GRG code for large sparse dynamic nonlinear optimization problems. Mathematical Programming, 31, 153-191. 
Drud, A. (1992). CONOPT - A large-scale GRG code. ORSA Journal on Computing, 6, 207-216.

Fallside, F., \& Perry, P. F. (1975). Hierarchical optimization of a water supply network. Proceedings of the IEEE, 122(2), 387-401.

GAMS (1997). The solver manuals. Washington, DC: GAMS Development Corporation.

Greco, M. (1997). WATERNET simulation users manual. Technical report WATERNET Project, ESPRIT-IV No. 22186.

Griñó, R. (1991). Neural networks for water demand time-series forecasting. In A. Prieto, Lecture Notes in Computer Science, vol. 540. Berlin: Springer.

Griño, R., \& Cembrano, G. (1991). Optimización en linea de estrategias de control de la red de distribución de agua abastecida por la $S G A B$ mediante la metodología de flujos sobre redes. Technical report No. IC-DT-1991.02 Instituto de Cibernética, Universidad Politécnica de Cataluña, Bercelona.

Isaacs, L. T., \& Mills, K. G. (1981). Linear theory methods for pipe network analysis. Journal of the Hydraulics Division ASCE, 9, 542-569.

Jeppson, R. W. (1983). Analysis of flow in pipe networks, (6th ed.) Ann Arbor Science, New York.

López, J., Cembrano, G., \& Cellier, F. (1996). Time series prediction using fuzzy inductive reasoning. Proceedings of the European simulation multiconference, ESM'96, San Diego: SCS International (pp. 765-770).
Minoux, M. (1986). Mathematical programming. Chichester, UK: Wiley. Murray, D. M., \& Yakowitz, S. J. (1979). Constrained differential dynamic programming and its application to multireservoir control. Water Resources Research, 15(5), 223-235.

Pérez, R., Cembrano, G., \& Quevedo, J. (1998). Identification of optimisation's tasks and criteria: Characterisation of SMAS network. Deliverable report No. 5.1, WATERNET ESPRIT IV Project No. 22.186, Barcelona.

Quevedo, J., Cembrano, G., Casanova, F., \& Ros, E. (1987). A contribution to the interactive dispatching of water distribution systems. Proceedings of IMACS international symposium on AI, expert systems and languages in modelling and simulation. Amsterdam: NorthHolland.

Quevedo, J., Cembrano, G., Gutiérrez, L., \& Valls, A. (1988). Timeseries modelling of water demand, study on short-term and longterm preditions. In B. Coulbeck, \& C. H. Orr, Computer applications of water supply and distribution. Lechworth, UK: Research Studies Press.

Solanas, J. L. (1974). Mando en tiempo real de un sistema de suministro de agua. Ph.D. thesis, Universidad Politécnica de Cataluña, Barcelona.

Solanas, J. L., \& Montoliu, J. M. (1988). Optimal operation of water systems. In B. Coulbeck, \& C. H. Orr, Computer applications of water supply and distribution. Lechworth, UK: Research Studies Press. 